\title{
A Flexible Superamphiphobic Film with 3D Conductive Network for Wearable Strain Sensors in Humid Conditions
}

\author{
Ya-Ru Ding, ${ }^{a}$ Chao-Hua Xue, ${ }^{* a b c}$ Xiao-Jing Guo, ${ }^{b}$ Xue Wang, ${ }^{b}$ Shun-Tian Jia, ${ }^{a}$ Qiu-Feng An ${ }^{d}$ \\ ${ }^{\text {a }}$ College of Bioresources Chemical and Materials Engineering, Shaanxi University of Science and \\ Technology, Xi'an 710021, China.
}

${ }^{\mathrm{b}}$ College of Materials Science and Engineering, Shaanxi University of Science and Technology, Xi'an 710021, China.

${ }^{\mathrm{c}}$ National Demonstration Center for Experimental Light Chemistry Engineering Education, Shaanxi University of Science and Technology, Xi'an 710021, China.

${ }^{\mathrm{d}}$ College of Chemistry and Chemical Engineering, Shaanxi University of Science and Technology, Xi'an 710021, China.

*Corresponding author. E-mail: xuechaohua@126.com.

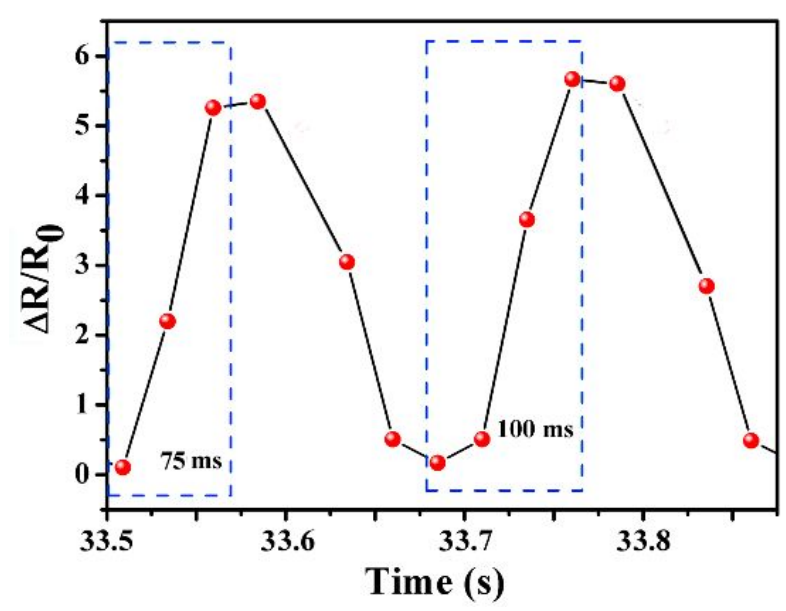

Fig. S1 The measurement of response and recovery time of strain sensor at a strain of $20 \%$. 

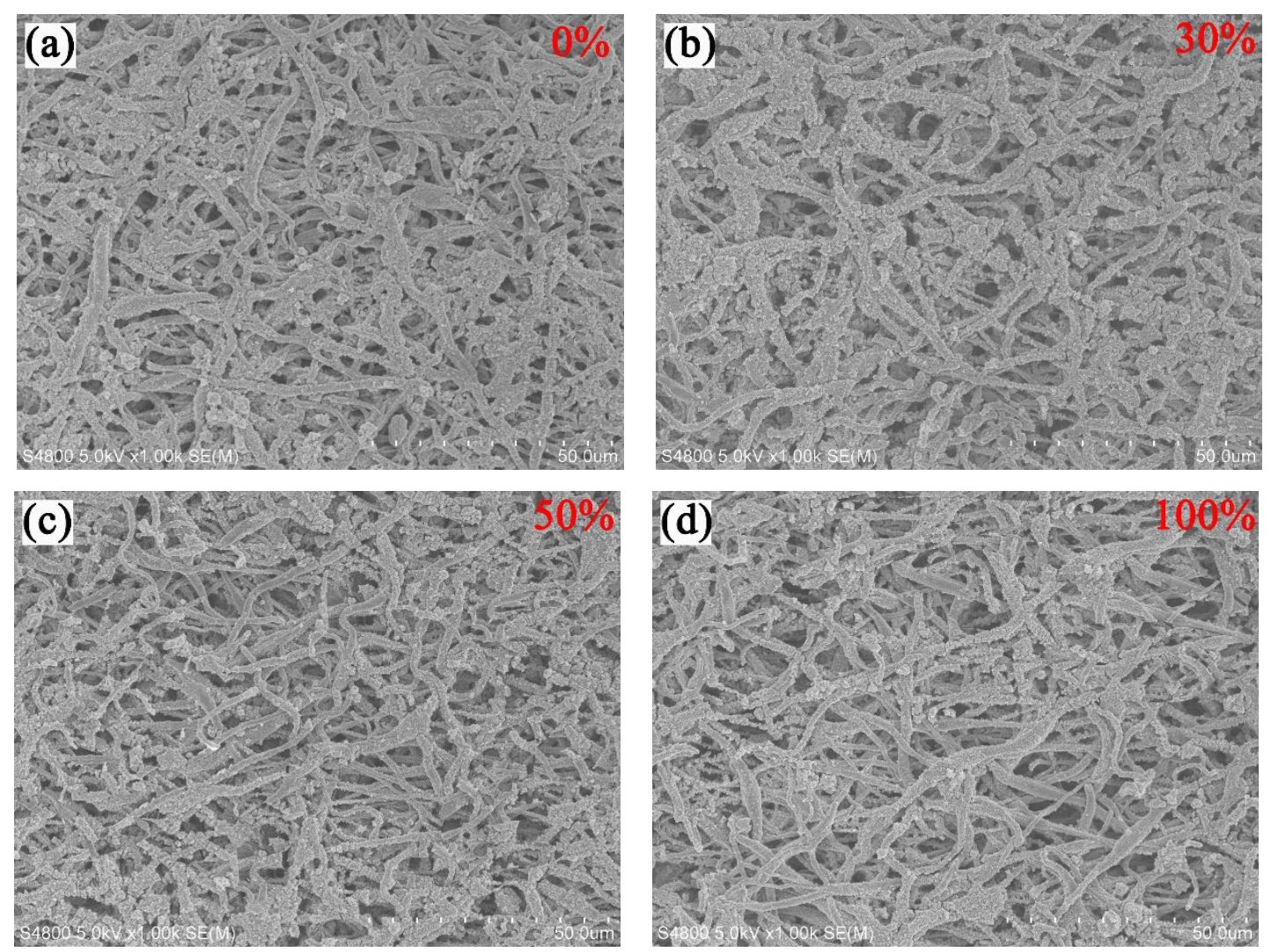

Fig. S2 SEM images of TPU/SCB conductive films with stretching at $0 \%$ (a), $30 \%$ (b), $50 \%$ (c), $100 \%(d)$. 

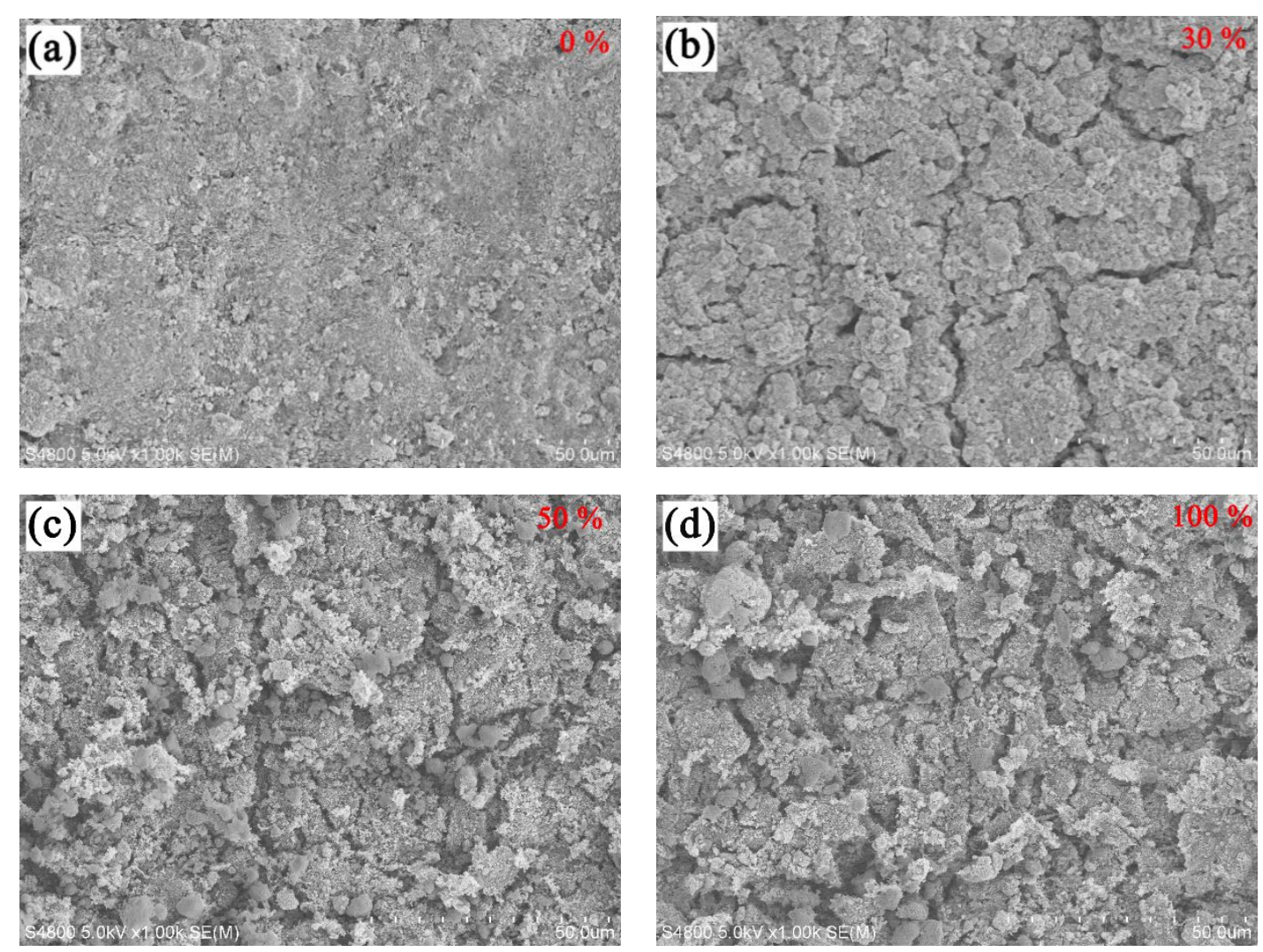

Fig. S3 SEM images of the $\mathrm{CNTs} / \mathrm{F}-\mathrm{SiO}_{2}$ coating on flexible TPU/SCB films with stretching at $0 \%$ (a), 30\% (b), 50\% (c), 100\% (d). 